\title{
Esquisse of the epidemiological scenario of chagas disease in Brazil.
}

\section{Brasileiro MR*}

Bachelor of Biological Sciences and Master of Animal Science Universidade do Oeste Paulista, UNOESTE, Brazil

\begin{abstract}
Through the data from the Ministry of Health / SVS - Notification of Injury Information System (SINAN), the positive cases reported from January 2001 to December 2014 were identified with the objective of obtaining a quantitative description of cases by Federal Unit ) And Region of the country. The analysis of the data resulted in the largest number of cases in the Northeast Region, with a percentage of $42.5 \%$ of the cases, out of a total of 3,827 cases reported in Brazil over a period of 14 years. It was possible to visualize an advance in the fight against Chagas' disease in the country, with a significant decrease in the number of annual cases of the regions, except for the North region that presented an increase in the annual incidence of cases.
\end{abstract}

Keywords: Chagas disease, American trypanosomiasis, Trypanosoma cruzi, Parasitic zoonosis.

Accepted on June 03, 2017

\section{Introduction}

Carlos Chagas, on a mission to control the outbreak of malaria in workers of the Central Railway of Brazil in 1907, became aware of a hematophagous insect, commonly known as a "barbeiro", which was described as a vector of the protozoan Trypanosoma cruzi (CHAGAS). Since then, studies have focused on the discovery of the infectious-contaminant aspects, control, way of life and other species of vectors and parasites that cause Chagas Disease. The causative agent of Chagas disease, T. cruzi flagellate protozoan belongs to the order Kinetoplastida, family Trypanosomatidae. Unlike other diseases transmitted by hematophagous vectors, the transmission does not occur by the inoculation of the agent with saliva but by the sting, followed by defecation in the act or after hematophagy, such feces contain infective forms of the parasite, which penetrate both in Wounds and mucous membranes, as well as through the bite hole of the vector itself [1]. Acute Chagas disease (ACD) or American trypanosomiasis is a unique anthropozoonosis in the Americas, representing the fourth major social impact among infectious and parasitic diseases in Latin America [2]. The vector transmission depends on three basic factors, being the presence of T. cruzi, domiciled triatomines and mammalian hosts in the home environment. This form of infection, called classical, affects $80 \%$ of infections, being directly linked to human factor and socioeconomic conditions [3]. Another route of infection is through blood transfusion, transplacental and organ transplantation, both of which involve an infected patient, and may also occur by accidental ingestion of triatomines or feces of the infected insect.

\section{Vector}

The "barber" is an arthropod of the class insecta, order hemiptera, family reduviidae and subfamily triatominae that feeds exclusively on homeothermic vertebrates, being called hematophagous. They are usually sheltered in a place very close to the source of food and can be found in the forest in birds' nests, animal scarf, tree trunk bark, wood fences and under stones, present average life between one and two years, With evolution of egg, nymph and adult. They have a great breeding capacity and, depending on the species, intense resistance to fasting.

\section{Life cycle}

Eggs: The female copulates once and after about 20 to 30 days begins the posture. Each female oviposes about 200 eggs. Egg hatching occurs on average 25 days after laying.

Nymphs: Eggs hatch into nymphs, which seek food 2 or 3 days after birth. After your first meal the nymph will suffer loss of your skin. The "barber" goes through 5 seedlings until reaching the adult stage.

Adults: The adult lives for a few months, reaching a year or more. The females do the first posture with about 2 months. After laying, they tend to migrate and form new colonies. The life cycle lasts on average from 1 to 2 years.

\section{Ecological distribution of triatomines}

A total 118 species of triatomines are known, and 105 are from the New World, both potential vectors for $T$. cruzi, being the most important vector in north-eastern Brazil, frequently found in wild and peridomiciliary habitats, where is strongly associated with shelters of goats, chickens and rocky ecotypes, and in the home environment it has birds and humans as the main food source.

\section{Clinic and Diagnosis}

The acute form of the disease usually occurs in children, with the presence of late chronic manifestations. The clinical manifestations are rare, being characterized by fever of variable intensity, malaise, lymphadenopathy and hepatosplenomegaly. 
Diagnosis in the acute phase is established by demonstration of the parasite through blood, culture or immunodiagnostic screening.

\section{Control}

Due to the infection transmission cycle, the control measures are generally directed to the control of the vector through chemical and biological control, and quality control of the blood transfused through the supervision of hemotherapy units in the control of quality of blood to be transfused through the serological screening of all blood donors with at least two sensitivity techniques. The main objective was to explain the epidemiological situation of Chagas' disease in Brazil from 2001 to 2014, resulting in the quantification of confirmed cases in the regions and identification of the regions with the highest percentage of cases.

\section{Materials and Methods}

The research was carried out without the approval of the Ethics Chamber in Animal Experimentation (CEUA), since it did not evaluate the use of animals, but rather a platform of the Ministry of Health / SVS-Information System of Notification Aggravations-SINAN online that allows the collection of tabulated and revised information [4]. With the collection of data on the platform, it was possible to identify the number of total cases in each year and each federative unit (UF), thus presenting the percentage of cases of the disease in the given period.

\section{Results and Discussion}

The Brazilian regions most affected by the disease were Northeast $(42.5 \%)$ and North $(41.3 \%)$, as shown in Table 1. Regarding the incidence of cases, it can be observed that in the period from 2001 to 2007 the Northeast region presented the highest number of cases in the country, and in the period from 2005 to 2014 there was a significant decrease in the number of cases in the region, which suggests a positive impact of control and awareness measures, supposedly adopted by the public health organs.

Table 1. Number of confirmed cases for ACD in Brazil-2001-2014.

\begin{tabular}{|c|c|c|c|c|c|c|}
\hline Ano/Região & Norte & Nordeste & Sudeste & Sul & Centro-Oeste & Total/Ano \\
\hline 2001 & 5 & 37 & 7 & 12 & 0 & 61 \\
\hline 2002 & 38 & 114 & 36 & 48 & 10 & 246 \\
\hline 2003 & 49 & 436 & 33 & 46 & 15 & 579 \\
\hline 2004 & 38 & 264 & 42 & 58 & 17 & 419 \\
\hline 2005 & 52 & 426 & 57 & 78 & 16 & 629 \\
\hline 2006 & 132 & 299 & 44 & 45 & 24 & 544 \\
\hline 2007 & 151 & 3 & 0 & 0 & 1 & 155 \\
\hline 2008 & 96 & 8 & 0 & 0 & 0 & 104 \\
\hline 2009 & 214 & 5 & 0 & 0 & 1 & 220 \\
\hline 2010 & 104 & 10 & 0 & 1 & 15 & 130 \\
\hline 2011 & 166 & 16 & 0 & 0 & 8 & 190 \\
\hline 2012 & 186 & 2 & 0 & 1 & 0 & 189 \\
\hline 2013 & 157 & 2 & 2 & 1 & 1 & 163 \\
\hline 2014 & 192 & 3 & 1 & 1 & 1 & 198 \\
\hline Total de Casos (\%) & $1580(41.3 \%)$ & $1625(42.5 \%)$ & $222(5.8 \%)$ & $291(7.6 \%)$ & $109(2.8 \%)$ & $3827(100 \%)$ \\
\hline Média de casos & 113/ano & 116/ano & 16/ano & 21/ano & 8/ano & 273/ano \\
\hline
\end{tabular}

Chagas disease is one of the main tropical diseases, its transmission is relatively simple, and having a wide variety of mammalian vectors and hosts. Knowing that vectors are included in the peridomiciliary environment, occupying areas of human activity, this fulfils the three requirements described by Dias in front of the line of infection and transmission. Since prevention and control measures adopted in the Northeast region during the period 2005 to 2014 have supposedly had a significant effect in relation to the number of cases, it is consistent with epidemiological research, with emphasis on both the quantitative description of cases and to monitor the evolution of the disease in the country $[5,6]$.

\section{Conclusion}

It was observed a high incidence in the number of cases of the Brazilian North region in relation to the other regions, which verified the second place in the total of cases of the country. On the other hand, there was significant control in the 
incidence of cases in other regions of the country over the years, mainly in the Northeast region. This suggests that measures of control and awareness applied to the population are essential for the prevention and combat of the disease.

\section{References}

1. Galvão C. Vectors of chagas disease in Brazil. Brazilian society of zoology, Curitiba, Brazil, 2014.

2. Schmunis GA, Dias JCP. Health sector reform, decentralization, prevention and control of vector-borne diseases. Cadernos de saúde pública 2000.

3. Dias JCP, Vinhaes MC, Silveira AC, et al. Priority research on Chagas' disease in the Amazon: shortmedium-term agenda. Journal of the Brazilian Society of Tropical Medicine 2001.
4. Ministry of health/SVS - Aacute chagas' disease: confirmed cases reported in the notification system information system-sinan-Brazil 2014.

5. Valente AS, Valente VC. Situation of chagas' disease in the amazon. Journal of the Brazilian society of tropical medicine 1993.

6. http://www.gbif.org/resource/81287.

\section{*Correspondence to}

Brasileiro MR

Bachelor of Biological Sciences and Master of Animal Science Universidade do Oeste Paulista UNOESTE 